\title{
Classical Theta Functions and Quantum Tori
}

\author{
By
}

\author{
Alan WeINSTEIN*
}

\begin{abstract}
The Schwartz kernel of the multiplication operation on a quantum torus is shown to be the distributional boundary value of a classical multivariate theta function. The kernel satisfies a Schrödinger equation in which the role of time is played by the deformation parameter $\hbar$ and the role of the hamiltonian by a Poisson structure. At least in some special cases, the kernel can be written as a sum of products of single variable theta functions.
\end{abstract}

A quantum torus is, by definition, a "space" whose (noncommutative) algebra of functions is obtained by deformation of the (commutative) algebra of functions on a torus $(\boldsymbol{R} / \boldsymbol{Z})^{d}$. In this note, we observe that the Schwartz kernels of the multiplication operators for a class of quantum tori are the (distributional) boundary values of classical theta functions, and we draw some conclusions from this observation. In particular, we show that each of these kernels satisfies a Schrödinger equation, in which the role of time is played by the deformation parameter $h$, and the role of the hamiltonian is played by the Poisson structure associated with the deformation. Thus, the "evolution in Plank's constant" of a torus is like the time evolution of a free particle, with the initial state (the Schwartz kernel of the operator of pointwise multiplication) being a delta function. like the initial state of a particle with certain position and totally uncertain momentum.

This evolution equation suggests a possible answer to the question of how a deformed product must behave after the deformation parameter $h$ has left the first infinitesimal neighborhood of zero.

We note that our work seems to be quite different from Manin's study [2] of quantum theta functions, which are special functions on the quantum tori themselves.

Communicated by T. Kawai, September 13, 1993.

1991 Mathematics Subject Classifications: 81S10, 33D10, 58B30.

* Department of Mathematics, University of California, Berkeley, CA 94720 USA.

Research partially supported by NSF Grant DMS-93-09653. 


\section{$\S 1$. The Kernel of Multiplication on a Quantum Torus}

The noncommutative multiplications on the space of functions on $\boldsymbol{T}^{d}$ which we will discuss in this paper are those which define the so called "noncommutative tori", or "quantum tori", an important class of examples of noncommutative differentiable manifolds, surveyed in [4]. Specifically, we consider the "star products" $*_{h}$ given in terms of the basis

$$
\left\{e_{m}(x)=e^{2 \pi i m x}\right\}_{m \in \boldsymbol{Z} d}
$$

of functions on $\boldsymbol{T}^{d}$ and the deformation parameter $h$ by

$$
e_{m} *_{\hbar} e_{n}=e^{-\pi i \hbar P(m n)} e_{m+n}
$$

where $P: \boldsymbol{R}^{d *} \times \boldsymbol{R}^{d *} \rightarrow \boldsymbol{R}$ is a skew-symmetric bilinear form.

Notice that $e_{m} *_{0} e_{n}=e_{m+n}$, which is the rule for ordinary (commutative) multiplication on $\boldsymbol{T}^{d}$, and

$$
\begin{aligned}
\frac{1}{i \hbar}\left(e_{m} *_{h} e_{n}-e_{n} *_{h} e_{m}\right) & =\frac{1}{i \hbar}\left(e^{-\pi i h P(m, n)}-e^{\pi i h P(m, n)} e_{m+n}\right. \\
& =-2 \pi P(m, n) e_{m+n}+\mathcal{O}(\hbar),
\end{aligned}
$$

so the Poisson structure which is the semiclassical limit of this deformation is given by

or, equivalently,

$$
\left\{e_{m}, e_{n}\right\}=-2 \pi P(m, n) e_{m+n},
$$

$$
\{f, g\}(x)=(1 / 2 \pi) P(d f(x), d g(x)) .
$$

The product defined by (1) is initially defined on the space $C^{\infty}\left(\boldsymbol{T}^{d}\right)$. The resulting algebra is denoted by $\mathcal{A}_{\hbar P}^{\infty}$ or by $C^{\infty}\left(\boldsymbol{T}_{h P}^{d}\right)$, where $\boldsymbol{T}_{h P}^{d}$ denotes the "quantum manifold" on which $\mathcal{A}_{\hbar P}^{\infty}$ is the algebra of "smooth functions".

Remark 1.1. The algebra $C\left(\boldsymbol{T}_{\hbar P}^{d}\right)$ of continuous functions on $\boldsymbol{T}_{\hbar P}^{d}$ is the $C^{*}$ algebra obtained by completing $\mathcal{A}_{\hbar P}^{\infty}$ with respect to a certain norm [4]. There seems to be no natural identification of the Banach space $C\left(\boldsymbol{T}_{h P}^{d}\right)$ with the space $C\left(\boldsymbol{T}^{d}\right)$ of continuous functions on the ordinary torus. As Rieffel points out in $[4]$, the elements of $C\left(\boldsymbol{T}_{h P}^{d}\right)$ are determined by their Fourier coefficients, but the set of possible Fourier series for these elements depends on $P$ and, just as in the case $P=0$, is difficult to describe in a simple way. The results in this paper may make it possible to explain the difference between $C\left(\boldsymbol{T}_{h P}^{d}\right)$ and $C\left(\boldsymbol{T}^{d}\right)$ in terms of the singular nature of the distribution kernel for multiplication in $C^{\infty}\left(\boldsymbol{T}_{h P}^{d}\right)$.

The product $*_{\hbar}$ for $\boldsymbol{T}^{d}$ may also be considered as the restriction to $C^{\infty}\left(\boldsymbol{T}^{d}\right)$ of the Moyal product 


$$
\left.\left(f *_{h} g\right)(x) \sim \sum_{j=0}^{\infty} \frac{1}{j !}\left[\frac{i \hbar}{4 \pi} P\left(\frac{\partial}{\partial y}, \frac{\partial}{\partial z}\right)\right]^{j}(f(y) g(z))\right|_{y=z=x}
$$

on $C^{\infty}\left(\boldsymbol{R}^{d}\right)$ (defining the quantum affine space $\boldsymbol{R}_{h P}^{d}$ ), where $C^{\infty}\left(\boldsymbol{T}^{d}\right)$ is identified with the space of $C^{\infty}$ functions on $\boldsymbol{R}^{d}$ invariant under translation by the lattice $\boldsymbol{Z}^{d}$.

When $P$ is nonsingular, the "inverse" of $P / 2 \pi$ is a symplectic structure on $T^{d}$ which we will denote by $\omega$. In this case, the product $*_{h}$ is also given by the integral formula

$$
\left(f *_{h} g\right)(x)=\int_{R^{d} \wedge_{R^{d}}(\pi \hbar)^{d}} e^{2 i(\omega(x-y \cdot x-z) / \hbar} f(y) g(z) d y d z .
$$

The improper integral in (2) can be defined as an oscillatory integral when $f$ and $g$ lie in the space of functions whose partial derivatives of all orders are each uniformly bounded, and the product belongs to the same space. (See [1] for a proof of the closure of this and other spaces under the Moyal product.) This fact and translation invariance allow the Moyal product to pass to $C^{\infty}\left(\boldsymbol{T}^{d}\right)$.

For singular $P$, the Moyal product can still be defined by an integral formula, but the kernel $K(x, y, z)=(\pi h)^{-d} e^{2 i \omega(x-y, x-z) / h}$ is no longer a smooth function. Instead, it is a delta-distribution supported by the set of triples $(x, y, z)$ for which the differences $x-y$ and $x-z$ (and hence $y-z$ as well) lie in the range of the map $\tilde{P}:\left(\boldsymbol{R}^{d}\right)^{*} \rightarrow \boldsymbol{R}^{d}$ associated with the bilinear form $P$, i. e. when $x, y$, and $z$ all lie in the same symplectic leaf of the Poisson structure $P$. We will see shortly that the product for $\boldsymbol{T}_{h P}^{d}$ is also given by a distribution kernel, which is singular even if $P$ is nondegenerate.

To find the kernel of multiplication on $\boldsymbol{T}_{h P}^{d}$, i.e., the distribution $K_{h P}(x, y, z)$ for which

$$
\left(f *_{\iota} g\right)(x)=\int_{T^{d}<_{T} d} K_{h P}(x, y, z) f(y) g(z) d y d z
$$

when $f, g \in C^{\infty}\left(\boldsymbol{T}^{d}\right)=C^{\infty}\left(\boldsymbol{T}_{h P}^{d}\right)$, we expand $f$ and $g$ in Fourier series:

$$
\begin{aligned}
& f(x)=\sum_{m} a_{m} e^{2 \pi \imath m x} \\
& 3(x)=\sum_{n} b_{n} e^{2 \pi \imath n x} .
\end{aligned}
$$

Then

$$
\begin{aligned}
\left(f *_{h} g\right)(x) & =\sum_{m, n \in \boldsymbol{Z}^{d}} a_{m} b_{n} e^{-\pi \imath h P(m, n)} e^{2 \pi i(m+n) \cdot x} \\
& =\sum_{m \in \boldsymbol{Z}^{d}} \int_{\boldsymbol{T}^{d}} e^{-2 \pi \imath m y} f(y) d y \int_{\boldsymbol{T}^{d}} e^{-2 \pi \imath n z} g(z) d z e^{-\pi i h P(m n)} e^{2 \pi i(m+n) x} \\
& =\int_{\boldsymbol{T}^{d} \boldsymbol{T}^{d}} \sum_{m \cdot n \in \boldsymbol{Z}^{d}} e^{-\pi \imath h P(m, n)-2 \pi i(m(y-x)+n(z-x))} f(y) g(z) d y d z
\end{aligned}
$$

where all quanticies are considered as distributions, so that the interchange of 
summation and integration is justified. Thus, the kernel of multiplication for our quantum torus $\boldsymbol{T}_{h P}^{d}$ is the distribution

$$
K_{h P}(x, y, z)=\sum_{m, n \in Z^{d}} e^{-\pi \imath h P(n, n)-2 \pi \imath(m(y-x)+n(z-x))} .
$$

Being translation invariant, the kernel can be written as $K_{h P}(x, y, z)=$ $L_{h P}(y-x, z-x)$, where

$$
L_{h P}(y, z)=\sum_{m, n \in Z^{d}} e^{-\pi i h P(m n)-2 \pi \imath(m y+n z)} .
$$

It is this "convolution kernel" $L_{h P}$, defined as a distribution on the group $\boldsymbol{T}^{d} \times \boldsymbol{T}^{d}$ by the sum (3), which we will analyze in the rest of this paper.

\section{§2. Differential Equation for the Kernel}

Differentiating the typical term in (3) by $h$ and writing $P(m, n)$ as a sum $\sum_{\jmath, k=1}^{d} P_{j k} m_{\jmath} n_{k}$, we find

$$
\begin{aligned}
\frac{d}{d h} e^{-\pi i h P(m, n)-2 \pi i(m y+n z)} & =-\pi i P(m, n) e^{-\pi i h P(m, n)-2 \pi i(n \imath y+n z)} \\
& =-\pi i \sum_{j, k=1}^{d} P_{j k} m_{\jmath} n_{k} e^{-\pi \imath h P(m, n)-2 \pi \imath(m y+n z)} \\
& =-\pi i P\left(\frac{1}{-2 \pi i} \frac{\partial}{\partial y}, \frac{1}{-2 \pi i} \frac{\partial}{\partial z}\right) e^{-\pi \imath h P(m, n)-2 \pi \imath(m y+n z)} .
\end{aligned}
$$

Applying this result to each term in (3) and adding, we find the "Schrödinger equation"

$$
i \frac{\hat{\partial}}{\partial \hbar} L_{h P}(y, z)=-\frac{1}{4 \pi} P\left(\frac{\partial}{\hat{\partial} y}, \frac{\partial}{\partial z}\right) L_{h P}(y, z) .
$$

Although $P$ is skew symmetric with respect to exchange of its two arguments, the operator $-(1 / 4 \pi) P(\partial / \partial y, \partial / \partial z)$ on $\boldsymbol{T}^{d} \times \boldsymbol{T}^{d}$ is self adjoint. It is the "quantum hamiltonian" corresponding to the classical hamiltonian, quadratic in the momenta, $p(y, \eta, z, \zeta)=(1 / 4 \pi) P(\eta, \zeta)$. If $P$ is nondegenerate, this hamiltonian is the "kinetic energy" and $-p(\partial / \partial y, \partial / \partial z)$ half the "laplacian" for a pseudoriemannian metric of signature $(d, d)$ on $\boldsymbol{T}^{d} \times \boldsymbol{T}^{d}=\boldsymbol{T}^{2 d}$. (A similar statement may be made when $P$ is degenerate, except that the metric is now "codegenerate".)

Finally, the "initial condition" for $L$, when $h=0$, is just the delta function $\delta(y, z)$ at the zero element of the group $\boldsymbol{T}^{d} \times \boldsymbol{T}^{d}$.

We may summarize the discussion above as follows:

The evolution in $h$ of the convolution kernel of multiplication for the quantum tori $\mathbb{T}_{\hbar P}^{d}$ is the same as the time evolution of a quantum "free particle" on the (ordinary) torus $\boldsymbol{T}^{2 d}$ with indefinite quadratic hamiltonian given by the Poisson 
structure $P / 2 \pi$. This particle is initially concentrated at the zero element of $Z^{2 d}$ but becomes completely nonlocalized as soon as $\hbar \neq 0$.

In other words, quantization appears to be a kind of "integration" of a bidifferential operator, the Poisson structure, to a 1-parameter family "group" in some sense ?) of Fourier bi-integral operators, the noncommutative multiplications.

\section{$\S 3$. The Quantization Kernel as a Theta Function}

The formula (3) exhibits the convolution kernel $L_{\hbar P}$ as a multidimensional theta function. Here, we should think of $(y, z)$ as a "single" variable in $\boldsymbol{R}^{2 \text { tz }}$ and $P(m, n)$ as a quadratic form in the variable $(m, n) \in Z^{2 d}$ (rather than $\left.\boldsymbol{Z}^{d} \times \boldsymbol{Z}^{d}\right)$. Since the imaginary part of the matrix $P(m, n)$ is zero, we are on the boundary of the region where the function $\vartheta(\vec{z}, \Omega)$ (see page 118 of [3]) is holomorphic.

In some special situations, we can diagonalize the quadratic form $P(m, n)$ by a $2 d \times 2 d$ matrix which nearly preserves the lattice $(2 \pi Z)^{2 d}$. This allows us to express $L_{h P}(y, z)$ in terms of theta functions of a single variable.

We will confine our attention to the simplest case $d=2$. In higher dimensions, interesting problems in the "symplectic geometry of numbers" should arise; we will not deal with them here.

In coordinates $\left(x_{1}, x_{2}\right)(\bmod \boldsymbol{Z})$ on $\boldsymbol{T}^{2}$, the most general translation-invariant Poisson structure is a constant multiple of $\partial / \partial x_{1} \wedge \partial / \partial x_{2}$. Since we already have the multiplier $h$ at our disposal, we will assume that $P=\partial / \partial x_{1} \wedge \partial / \partial x_{2}$. Then $\tilde{P}\left(d x_{1}\right)=\partial / \partial x_{2}$ and $\tilde{P}\left(d x_{2}\right)=-\partial / \partial x_{1}$, so $\tilde{P}$ is represented with respect to the standard bases of tangent and cotangent vectors by the matrix $\left(\begin{array}{lr}0 & -1 \\ 1 & 0\end{array}\right)$.

If we denote the coordinates on $\boldsymbol{T}^{2} \times \boldsymbol{T}^{2}$ by $\left(y_{1}, y_{2}, z_{1}, z_{2}\right)$ and the corresponding coordinates on $\boldsymbol{R}^{2 *} \times \boldsymbol{R}^{2 *}$ by $\left(m_{1}, m_{2}, n_{1}, n_{2}\right)$, then the quadratic form $P(m, n)$ is $m_{1} n_{2}-m_{2} n_{1}$, and the convolution kernel for multiplication is

$$
L_{\hbar P}(y, z)=\sum_{\left(m_{1}, n_{1}, m_{2}, n_{2}\right) \in \boldsymbol{Z}^{d^{2}}} e^{-\pi i \hbar\left(m_{1} n_{2}-m_{2} n_{1}\right)-2 \pi i\left(n_{1} y_{1}+m_{2} y_{2}+n_{1} z_{1}+n_{2} z_{2}\right)} .
$$

To diagonalize the quadratic form $P(m, n)$, we introduce the coordinates $u_{1}=m_{1}+n_{2}, v_{1}=m_{1}-n_{2}, u_{2}=m_{2}-n_{1}, v_{2}=m_{2}+n_{1}$. The inverse transformation is $m_{1}=(1 / 2)\left(u_{1}+v_{1}\right), \quad n_{1}=(1 / 2)\left(v_{2}-u_{2}\right), \quad m_{2}=(1 / 2)\left(u_{2}+v_{2}\right), \quad n_{2}=(1 / 2)\left(u_{1}-v_{1}\right) . \quad$ Integer values of $\left(m_{1}, m_{2}, n_{1}, n_{2}\right)$ correspond to integer values of $\left(u_{1}, u_{2}, v_{1}, v_{2}\right)$ for which $u_{1} \pm v_{1}$ and $u_{2} \pm v_{2}$ are even, conditions which define a sublattice $\Lambda \times \Lambda$ of $Z^{2} \times Z^{2}$. Now we have 


$$
\begin{gathered}
L_{\hbar P}(y, z)=\sum_{\left(u_{1}, v_{1}, u_{2}, v_{2}\right) \in \Lambda \wedge A} e^{(-\pi i \hbar / 4)\left(u_{1}^{2}-v_{1}^{2}+u_{2}^{2}-v_{2}^{2}\right)-\pi i\left(u_{1}\left(y_{1}+z_{2}\right)+u_{2}\left(y_{2}-z_{1}\right)+v_{1}\left(y_{1}-z_{2}\right)+v_{2}\left(y_{2}+z_{1}\right)\right)} \\
=\sum_{\left(u_{1}, v_{1}, u_{2}, v_{2}\right) \in \Lambda A} e^{-\pi i \hbar u_{1}^{2} / 4-\pi i u_{1}\left(y_{1}+z_{1}\right)} e^{\pi i \hbar v_{1}^{2} / 4-\pi i v_{1}\left(y_{1}-z_{2}\right)} \\
\times e^{-\pi i \hbar u_{2}^{2} / 4-\pi i u_{2}\left(y_{2}-z_{1}\right)} e^{\pi i \hbar v_{2}^{2} / 4-\pi i v_{2}\left(y_{2}+z_{1}\right)} .
\end{gathered}
$$

We would have succeeded by the last expression in splitting $L_{h P}(y, z)$ into a product of single variable theta functions were it not for the fact that the lattice $\Lambda \times \Lambda$ makes the variables $\left(u_{1}, v_{1}, u_{2}, v_{2}\right)$ dependent on one another.

Still, things are not so bad, since $\left(u_{j}, v_{j}\right)$ belongs to $\Lambda$ if and only if $u_{j}$ and $v_{j}$ are either both even or both odd. Thus, $\Lambda$ is the union of $2 \boldsymbol{Z} \times 2 \boldsymbol{Z}$ and $(2 \boldsymbol{Z}+1) \times(2 \boldsymbol{Z}+1)$, and we can write $L_{\hbar P}(y, z)$, with all sums over $\boldsymbol{Z}$, as

$$
\begin{aligned}
& \left(\sum_{n} e^{-\pi i \hbar n^{2}-2 \pi i n\left(y_{1}+z_{2}\right)} \sum_{n} e^{\pi i \hbar n^{2}-2 \pi i n\left(y_{1}-z_{2}\right)}\right. \\
& \left.+\sum_{n} e^{-\pi i h(n+1 / 2)^{2}-2 \pi i(n+1 / 2)\left(y_{1}+z_{2}\right)} \sum_{n} e^{\pi i \hbar(n+1 / 2)^{2}-2 \pi i(n+1 / 2)\left(y_{1}-z_{2}\right)}\right) \\
& \quad \times\left(\sum_{n} e^{-\pi i \hbar n^{2}-2 \pi i n\left(y_{2}-z_{1}\right)} \sum_{n} e^{\pi i \hbar n^{2}-2 \pi i n\left(y_{2}+z_{1}\right)}\right. \\
& \left.+\sum_{n} e^{-\pi i \hbar(n+1 / 2)^{2}-2 \pi i(n+1 / 2)\left(y_{2}-z_{1}\right)} \sum_{n} e^{\pi i \hbar(n+1 / 2)^{2}-2 \pi i(n+1 / 2)\left(y_{2}+z_{1}\right)}\right) .
\end{aligned}
$$

Using the "half-integer theta functions" and some of the simple theta identities given in Section I.4 of [3] (see especially page 17), we can write:

$$
\begin{aligned}
L_{\hbar P}(y, z)= & \left.\vartheta_{00}\left(y_{1}+z_{2},-\hbar\right) \vartheta_{00}\left(y_{1}-z_{2}, \hbar\right)+\vartheta_{10}\left(y_{1}+z_{2},-\hbar\right) \vartheta_{10}\left(y_{1}-z_{2}, \hbar\right)\right) \\
& \times\left(\vartheta_{00}\left(y_{2}-z_{1},-\hbar\right) \vartheta_{00}\left(y_{2}+z_{1}, \hbar\right)+\vartheta_{10}\left(y_{2}-z_{1},-\hbar\right) \vartheta_{10}\left(y_{2}+z_{1}, \hbar\right)\right) .
\end{aligned}
$$

Alternatively, we can express everything in terms of the basic theta function

$$
\vartheta(z, \tau)=\sum_{n \in Z} e^{\pi i n^{2} \tau+2 \pi i n z}
$$

as:

(5) $L_{\pi P}(y, z)$

$$
\begin{aligned}
= & \left(\vartheta\left(y_{1}+z_{2},-\hbar\right) \vartheta\left(y_{1}-z_{2}, h\right)+e^{-2 \pi i y_{1}} \vartheta\left(y_{1}+z_{2}+\frac{1}{2} \hbar,-\hbar\right) \vartheta\left(y_{1}-z_{2}-\frac{1}{2} h, \hbar\right)\right) \\
& \times\left(\vartheta\left(y_{2}-z_{1},-\hbar\right) \vartheta\left(y_{2}+z_{1}, h\right)+e^{-2 \pi i y_{2}} \vartheta\left(y_{2}-z_{1}+\frac{1}{2} h-, \hbar\right) \vartheta\left(y_{2}+z_{1}-\frac{1}{2} h, \hbar\right)\right) .
\end{aligned}
$$

\section{$\S 4$. Discussion}

J. J. Duistermaat has pointed out that, although the theta function (distribution) can be continued from the real axis to a complex half plane, the pre- 
sence in (5) of products in which the theta function is evaluated at both $\hbar$ and $-\hbar$ shows that the kernel $L_{\hbar P}$ itself admits no such continuation. We hope that a better understanding of the analytic properties of $L$ can come from its expression in terms of theta functions, not only in the simple case $d=2$ considered above, but also in general, where there may always be a local decomposition similar to (5).

On the other hand, our work suggests the possibility of something even more interesting-the application of the extensive $C *$-algebraic theory of quantum tori (once again, we cite [4] for a survey of this theory) to derive new results, or reinterpret old ones, about the classical theta functions. Note that the associativity of the $*_{\hbar}$ product becomes an (integral) identity for the theta functions. Is it interesting? Is it known?

A symplectic geometric construction of quantum tori was given in [5]. The symplectic groupoid structures on $T^{*} \boldsymbol{T}^{d}$ described in that paper may be interpreted as the wavefront sets (or, more properly, "frequency sets", since asymptotics in $h$ are involved) of the kernels discussed here.

Finally, we return to the Schrödinger equation

$$
\left(i \frac{\partial}{\partial \hbar}+\frac{1}{4 \pi} P\left(\frac{\partial}{\partial y}, \frac{\partial}{\partial z}\right)\right) K_{h}(x, y, z)=0
$$

for which $K$ is the "fundamental solution" in the sense that $K_{0}(x, y, z)=$ $\delta(y-x, z-x)$. Do all "interesting" quantizations satisfy an equation of this nature? (The Moyal quantization of $\boldsymbol{R}^{d}$ does.) Finding such an equation would be an important step toward determining the "natural" deformation quantizations of given Poisson structures.

A major obstacle to extending the Schrödinger equation to the general case is that, in the expression $P(\partial / \partial y, \partial / \partial z)$, the vectors $\partial / \partial y$ and $\partial / \partial z$ may live at different points. There are, however, some interesting quantizations (notably, the case of quantum groups), of manifolds with Poisson structures admitting a simple expression in terms of globally defined vector fields.

\section{References}

[1] Hörmander, L., The Weyl calculus of pseudo-differential operators, Comm. Pure Appl. Math., 32 (1979), 359-443.

[2] Manin, Y.I., Quantized theta functions, Prog. Theor. Phys. Suppl., 102 (1990), 219-228.

[3] Mumford, D., Tata Lectures on Theta I, Birkhäuser, Boston, 1983.

[4] Rieffel, M. A., Non-commutative tori-a case study of noncommutative differentiable manifolds, Contemporary Math., 105 (1990), 191-211.

[5] Weinstein, A., Symplectic groupoids, geometric quantization, and irrational rotation algebras, Symplectic geometry, groupoids, and integrable systems, Séminaire sud-Rhodanien de géométrie à Berkeley (1989), P. Dazord and A. Weinstein, eds., Springer-MSRI Series (1991), 281-290. 
